\title{
Synthesis of 1-benzoxepin-5-ones (ols) from salicylaldehydes via ring-closing metathesis
}

\author{
Sie-Rong Li, ${ }^{\text {a,b }}$ Hsing-Ming Chen, ${ }^{c}$ Liang-Yeu Chen, ${ }^{\text {b Jui-Chi Tsai, }}$ Po-Yuan Chen, \\ Sodio Chih-Neng Hsu, ${ }^{a}$ and Eng-Chi Wang ${ }^{a^{*}}$ \\ ${ }^{a}$ Faculty of Medicinal and Applied Chemistry, Kaohsiung Medical University, Kaohsiung City, \\ Taiwan 807 \\ ${ }^{b}$ Institute of Pharmaceutical Sciences, Kaohsiung Medical University, Kaohsiung City, Taiwan \\ 807 \\ ${ }^{c}$ School of Medical and Health Sciences, Fooyin University, Kaohsiung County, Taiwan 831 \\ E-mail: enchwa@,kmu.edu.tw
}

\begin{abstract}
A new synthetic method for 1-benzoxepin-5-ones and 1-benzoxepin-5-ols from salicylaldehydes was described. Based on $O$-allylation, Grignard reaction, oxidation, and ring-closing metathesis in sequence, salicylaldehydes were converted to the target compounds in good yields, respectively.
\end{abstract}

Keywords: Ring-closing metathesis, salicylaldehydes, 1-benzoxepin-5-ones, 1-benzoxepin-5-ols

\section{Introduction}

The 1-benzoxepine moiety which plays a core structure both in naturally occurring products ${ }^{1}$ and in certain synthetic biological molecules, ${ }^{2}$ have abstracted the attention of chemists. In addition, benzoxepinone which has been employed as starting material, can be converted to corresponding quinoline by the Friedlander reaction, ${ }^{3}$ and can be transformed into benzoxepine by isomerization of double bond, reduction of carbonyl group, and dehydration of giving alcohol in sequence. ${ }^{4}$ The major synthetic methods for benzoxepinones which were reported in literatures, include the cyclopropanation and sequential reductive cleavage of flavones, ${ }^{5}$ the reaction of bromoalkyl ketones through the sequential reduction and oxidation, ${ }^{6}$ the reaction of dihydrobenzoxepinone via silylation and following by desilylation with DDQ and collidine. ${ }^{7}$ The drawbacks of those reported methods include the lack of conciseness, straightforward, and commercial available starting materials. Furthermore, the synthesis of benzoxepin-5-ols was paid little attention in reported literatures. ${ }^{4,8}$ Therefore, to develop a concise and practical method for the title compounds is requisite and significant. Since Grubbs' catalyst was developed in 1995, 
the ring-closing metathesis (RCM) has been widely applied to the compounds which were difficulty to be synthesized by the previous reported methods. ${ }^{9}$ However, the synthesis of 1benzoxepin-5-ols and 1-benzoxepin-5-ones utilized RCM has not been reported in current publications. Based on the chemistry of RCM, herein we would like to report a concise and practical method for those compounds (Scheme 1).

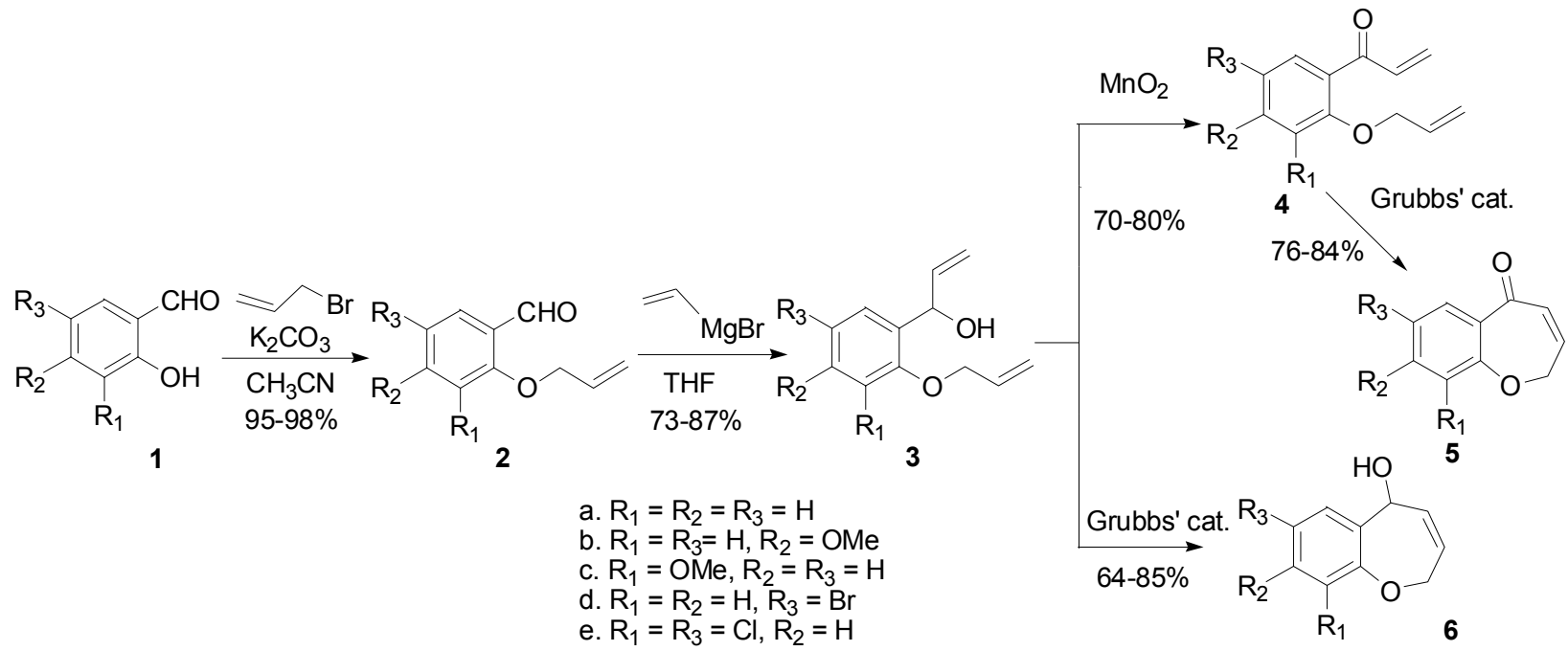

\section{Scheme 1}

\section{Results and Discussion}

By the reaction of salicylaldehydes (1a-e) and allyl bromide in the presence of potassium carbonate in refluxing acetonitrile for $4 \mathrm{~h}$, allyloxybenzaldehydes (2a-e) was obtained in 95-98\% yields. At the same condition if the reaction was carried out in refluxing acetone instead of acetonitrile, the yield of $\mathbf{2 a - e}$ was decreased. ${ }^{10}$ Subsequently, 2a-e was reacted with vinylmagnesium bromide to give (2-allyloxyaryl)-2-propen-1-ols (3a-e) in 73-87\% yields, respectively. The giving 3a-e which are all new compounds except 3c, have satisfactory spectral data. Followed by oxidation of 3a-e with $\mathrm{MnO}_{2}$ in dichloromethane, (2-allyloxyaryl)-2-propen-1ones (4a-e) were obtained in $70-80 \%$ yields, respectively, together with small amount of unidentified by-product. The products 4a-e which are all new compounds except $\mathbf{4 c}$, have satisfactory spectral data. Subsequently, by the treatment of 4a-e with Grubbs catalyst $\left(2^{\text {nd }}\right.$ generation) in dichloromethane at room temperature for $6 \mathrm{hr}, 2 \mathrm{H}$-1-benzoxepin-5-ones (5a-e) were produced in 76-84\% yields, respectively. Furthermore, by the treatment of (2-allyloxyaryl)2-propen-1-ol (3a-e) with Grubbs catalyst $\left(2^{\text {nd }}\right.$ generation) in dichloromethane at room temperature for $6 \mathrm{hr}, 2 \mathrm{H}$-1-benzoxepin-5-ol (6a-e) were given in 64-85\% yields, respectively. Thus, we have established a new route to both $2 H$-1-benzoxepin-5-ones and $2 H$-1-benzoxepin-5ols. The structure of 5a-e and 6a-e were respectively elucidated by spectral data such as ${ }^{1} \mathrm{H}$ - 
NMR, ${ }^{13} \mathrm{C}-\mathrm{NMR}$ and mass spectra. The typical signals of ${ }^{1} \mathrm{H}-\mathrm{NMR}$ of $2 \mathrm{H}$-1-benzoxepin-5-ones (5a-e) and 2H-1-benzoxepin-5-ols (6a-e), such as H-2, H-3, H-4, and H-5, together with typical carbonyl carbon of ${ }^{13} \mathrm{C}$-NMR of 5a-e were summarized in Table 1.

Table 1. The typical signals of ${ }^{1} \mathrm{H}-\mathrm{NMR}$ of $2 H$-1-benzoxepin-5-one (5a-e) and $2 H$-1-benzooxepin-5-ol (6a-c)
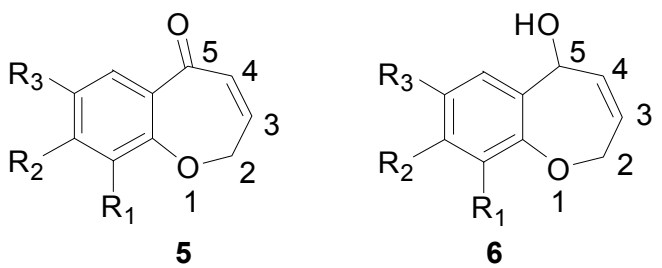

a. $R_{1}=R_{2}=R_{3}=H$
b. $R_{1}=R_{3}=H, R_{2}=O M e$
c. $R_{1}=O M e, R_{2}=R_{3}=H$
d. $R_{1}=R_{2}=H, R_{3}=B r$
e. $R_{1}=R_{3}=C l, R_{2}=H$

6

\begin{tabular}{cccccc}
\hline Compound & H-2 & H-3 & H-4 & H-5 & C-5 $^{\text {a }}$ \\
\hline $\mathbf{5 a}$ & $4.55(\mathrm{dd})$ & $6.75(\mathrm{dt})$ & $6.43(\mathrm{dt})$ & - & 189.86 \\
$\mathbf{5 b}$ & $4.68(\mathrm{dd})$ & $6.69(\mathrm{dt})$ & $6.41(\mathrm{dt})$ & - & 188.14 \\
$\mathbf{5 c}$ & $4.78(\mathrm{dd})$ & $6.76(\mathrm{dt})$ & $6.44(\mathrm{dt})$ & - & 190.37 \\
$\mathbf{5 d}$ & $4.73(\mathrm{dd})$ & $6.78(\mathrm{dt})$ & $6.41(\mathrm{dt})$ & - & 188.22 \\
$\mathbf{5 e}$ & $4.81(\mathrm{dd})$ & $6.80(\mathrm{dt})$ & $6.41(\mathrm{dt})$ & - & 187.89 \\
$\mathbf{6 a}$ & $4.52,4.60(\mathrm{ddd})$ & $5.48(\mathrm{~m})$ & $5.99(\mathrm{ddt})$ & $5.50(\mathrm{~m})$ & - \\
$\mathbf{6 b}$ & $4.48,4.57(\mathrm{ddd})$ & $5.46(\mathrm{~m})$ & $5.97(\mathrm{ddt})$ & $5.38(\mathrm{~m})$ & - \\
$\mathbf{6 c}$ & $4.52,4.59(\mathrm{ddd})$ & $5.47(\mathrm{~m})$ & $5.97(\mathrm{ddt})$ & $5.54(\mathrm{~m})$ & - \\
$\mathbf{6 d}$ & $4.46,4.63(\mathrm{ddd})$ & $5.48(\mathrm{~m})$ & $5.92(\mathrm{ddt})$ & $5.58(\mathrm{~m})$ & - \\
$\mathbf{6 e}$ & $4.47,4.70(\mathrm{ddd})$ & $5.46(\mathrm{~m})$ & $5.89(\mathrm{ddt})$ & $5.69(\mathrm{~m})$ & - \\
\hline
\end{tabular}

${ }^{\mathrm{a}}$ The chemical shifts of carbonyl carbon (C-5) of 5a-e in ${ }^{13} \mathrm{C}-\mathrm{NMR}$ spectra.

In conclusion, a new synthetic method for benzoxepinones and benzoxepinols from salicylaldehydes was established. The application of $\alpha, \beta$-unsaturated carbonyl functionality of benzoxepinones and allyl alcoholic functionality of benzoxepinols to synthesize some related compounds is in progressive.

\section{Experimental Section}

General Procedures. Melting points (Yanaco micro melting-point apparatus) were uncorrected. ${ }^{1} \mathrm{H}-\mathrm{NMR}$ and ${ }^{13} \mathrm{C}$-NMR spectra were obtained on a Varian Gemini-200 or Varian Unity plus 400 Spectrometer. Chemical shifts were measured in parts per million with respect to TMS. Mass spectra were recorded on a Chem/hp/middle spectrometer connected to a Hewlett Packard series II model gas-liquid chromatograph. HRMS spectra were performed on a JEOL JMS SX/SX 102A instrument. Silica gel (230-400 mesh) for column chromatography and precoated silica gel 
plates (60 F-254) for TLC was purchased from E. Merck Company. UV light (254 nm) was used to detect spots on TLC plates after development.

\section{General procedure for the preparation of 2-allyloxybenzaldehydes (2a-e)}

The 2-hydroxybenzaldehydes (1a-e) $(100 \mathrm{mmol})$ dissolved in anhydrous acetonitrile $(150 \mathrm{~mL})$ was added anhydrous $\mathrm{K}_{2} \mathrm{CO}_{3}(120 \mathrm{mmol})$. The mixture which was obtained was stirred and added allyl bromide $(120 \mathrm{mmol})$ and heated to reflux for $4 \mathrm{hr}$. Work-up as in the general procedure gave crude 2a-e which was further purified by silica-gel column (ethyl acetate: $n$ hexane $=1: 15)$ to give pure $\mathbf{2 a - e}$.

2-Allyloxybenzaldehyde (2a). ${ }^{\mathbf{1 0}}$ (15.69 $\left.\mathrm{g}, 97 \%\right)$ was obtained as colorless liquid, $\mathrm{R}_{\mathrm{f}}=0.16$ (ethyl acetate: $n$-hexane $=1: 15),{ }^{1} \mathrm{H}-\mathrm{NMR}\left(\mathrm{CDCl}_{3}, 400 \mathrm{MHz}\right) \delta 4.65(\mathrm{dt}, J=5.2,1.2 \mathrm{~Hz}, 2 \mathrm{H}$, $\mathrm{OC}_{2}{ }_{2} \mathrm{CH}=\mathrm{CH}_{2}$ ), 5.34 (ddt, $J=10.4,1.2,1.2 \mathrm{~Hz}, 1 \mathrm{H}, \mathrm{OCH}_{2} \mathrm{CH}=\mathrm{CH}_{\mathrm{a}} \mathrm{H}_{\mathrm{b}}$ ), 5.45 (ddt, $J=17.2,1.2$, $\left.1.2 \mathrm{~Hz}, 1 \mathrm{H}, \mathrm{OCH}_{2} \mathrm{CH}=\mathrm{CH}_{a} \underline{H}_{b}\right), 6.01$ (ddt, $J=17.2,10.4,5.2 \mathrm{~Hz}, 1 \mathrm{H}, \mathrm{OCH}_{2} \mathrm{C} \underline{H}=\mathrm{CH}_{2}$ ), 6.97 (m, 1H, ArH), 7.02 (m, 1H, ArH), 7.52 (m, 1H, ArH), 7.83 (m, 1H, ArH), 10.53 (s, 1H, CHO); ${ }^{13} \mathrm{C}-$ NMR $\left(\mathrm{CDCl}_{3}, 100 \mathrm{MHz}\right) \delta 69.0,112.8,117.9,120.7,125.0,128.3,132.3,135.8,160.8,189.6$; EI-MS (70 eV) m/z (rel. intensity, \%) $163\left([\mathrm{M}+1]^{+}, 100\right), 162\left(\mathrm{M}^{+}, 24\right), 161$ (54), 133 (24), 121 (76), 120 (19), 105 (18), 92 (26); HRMS calcd for $\mathrm{C}_{10} \mathrm{H}_{10} \mathrm{O}_{2}$ : 162.0681. Found: 162.0680.

2-Allyloxy-4-methoxybenzaldehyde (2b). ${ }^{\mathbf{1 1}}$ (18.24 g, 95\%) was obtained as colorless crystal, mp 37-38 ${ }^{\circ} \mathrm{C}, \mathrm{R}_{\mathrm{f}}=0.33$ (ethyl acetate: $n$-hexane $\left.=1: 6\right),{ }^{1} \mathrm{H}-\mathrm{NMR}\left(\mathrm{CDCl}_{3}, 200 \mathrm{MHz}\right) \delta 3.85(\mathrm{~s}$, $3 \mathrm{H}, \mathrm{OCH}_{3}$ ), 4.62 (dt, $J=5.2,1.4 \mathrm{~Hz}, 2 \mathrm{H}, \mathrm{OC}_{2} \mathrm{CH}_{2} \mathrm{CH}_{2}$ ), 5.33 (ddt, $J=10.6,1.4,1.4 \mathrm{~Hz}, 1 \mathrm{H}$, $\mathrm{OCH}_{2} \mathrm{CH}=\mathrm{C}_{\mathrm{a}} \mathrm{H}_{\mathrm{b}}$ ), 5.45 (ddt, $J=17.4,1.4,1.4 \mathrm{~Hz}, 1 \mathrm{H}, \mathrm{OCH}_{2} \mathrm{CH}=\mathrm{CH}_{\mathrm{a}} \underline{\mathrm{H}}_{\mathrm{b}}$ ), 6.04 (ddt, $J=17.4$, 10.6, $\left.5.2 \mathrm{~Hz}, 1 \mathrm{H}, \mathrm{OCH}_{2} \mathrm{CH}=\mathrm{CH}_{2}\right), 6.43(\mathrm{~d}, J=2.0 \mathrm{~Hz}, 1 \mathrm{H}, \mathrm{ArH}), 6.54(\mathrm{dd}, J=8.8,2.0 \mathrm{~Hz}, 1 \mathrm{H}$, $\operatorname{ArH}), 7.81(\mathrm{~d}, J=8.8 \mathrm{~Hz}, 1 \mathrm{H}, \mathrm{ArH}), 10.35(\mathrm{~s}, 1 \mathrm{H}, \mathrm{CHO}) ;{ }^{13} \mathrm{C}-\mathrm{NMR}\left(\mathrm{CDCl}_{3}, 50 \mathrm{MHz}\right) \delta 55.6$, 69.1, 99.0, 106.0, 118.1, 119.2, 130.4, 132.2, 162.6, 166.0, 188.2; EI-MS (70 eV) m/z (rel. intensity, \%) $193\left([\mathrm{M}+1]^{+}, 30\right), 192\left(\mathrm{M}^{+}, 35\right), 164$ (28), 163 (54), 151 (100), 150 (91), 135 (45), 122 (27), 95 (28); HRMS calcd for $\mathrm{C}_{11} \mathrm{H}_{12} \mathrm{O}_{3}$ : 192.0786. Found: 192.0784.

2-Allyloxy-3-methoxybenzaldehyde (2c). ${ }^{\mathbf{1 2}}(18.82 \mathrm{~g}, 98 \%)$ was obtained as colorless liquid, $\mathrm{R}_{\mathrm{f}}$ $=0.26$ (ethyl acetate: $n$-hexane $=1: 6),{ }^{1} \mathrm{H}-\mathrm{NMR}\left(\mathrm{CDCl}_{3}, 200 \mathrm{MHz}\right) \delta 3.86\left(\mathrm{~s}, 3 \mathrm{H}, \mathrm{OCH}_{3}\right), 4.62$ $\left(\mathrm{dt}, J=6.0,1.2 \mathrm{~Hz}, 2 \mathrm{H}, \mathrm{OC}_{2}{ }_{2} \mathrm{CH}=\mathrm{CH}_{2}\right.$ ), 5.33 (ddt, $J=10.2,1.2,1.2 \mathrm{~Hz}, 1 \mathrm{H}, \mathrm{OCH}_{2} \mathrm{CH}=\mathrm{CH}_{\mathrm{a}} \mathrm{H}_{\mathrm{b}}$ ), 5.45 (ddt, $J=17.4,1.2,1.2 \mathrm{~Hz}, 1 \mathrm{H}, \mathrm{OCH}_{2} \mathrm{CH}=\mathrm{CH}_{\mathrm{a}} \underline{\mathrm{H}}_{\mathrm{b}}$ ), 6.04 (ddt, $J=17.4,10.2,6.0 \mathrm{~Hz}, 1 \mathrm{H}$, $\left.\mathrm{OCH}_{2} \mathrm{C} \underline{\mathrm{H}}=\mathrm{CH}_{2}\right), 7.10$ (m, 2H, ArH), 7.37 (m, 1H, ArH), $10.41(\mathrm{~s}, 1 \mathrm{H}, \mathrm{CHO}) ;{ }^{13} \mathrm{C}-\mathrm{NMR}\left(\mathrm{CDCl}_{3}\right.$, $50 \mathrm{MHz}) \delta 55.9,75.0,113.3,117.9,118.9,124.0,130.0,133.0,151.1,152.9,190.2$; EI-MS (70 eV) $m / z$ (rel. intensity, \%) 193 ([M+1] $\left.]^{+}, 100\right), 192\left(\mathrm{M}^{+}, 54\right), 175$ (17), 166 (20), 164 (29), 163 (55), 151 (83), 136 (17) , 131 (20), 122 (20); HRMS calcd for $\mathrm{C}_{11} \mathrm{H}_{12} \mathrm{O}_{3}$ : 192.0786. Found: 192.0783 .

2-Allyloxy-5-bromobenzaldehyde (2d). ${ }^{13}(23.03 \mathrm{~g}, 96 \%)$ was obtained as colorless liquid, $\mathrm{R}_{\mathrm{f}}=$ 0.50 (ethyl acetate: $n$-hexane $=1: 9),{ }^{1} \mathrm{H}-\mathrm{NMR}\left(\mathrm{CDCl}_{3}, 400 \mathrm{MHz}\right) \delta 4.60(\mathrm{dt}, J=5.2,1.6 \mathrm{~Hz}$, $2 \mathrm{H}, \mathrm{OC}_{2}{ }_{2} \mathrm{CH}=\mathrm{CH}_{2}$ ), 5.31 (ddt, $\left.J=10.8,1.6,1.6 \mathrm{~Hz}, 1 \mathrm{H}, \mathrm{OCH}_{2} \mathrm{CH}=\mathrm{C}_{\mathrm{H}} \mathrm{H}_{\mathrm{b}}\right), 5.40$ (ddt, $J=17.2$, 1.6, $1.6 \mathrm{~Hz}, 1 \mathrm{H}, \mathrm{OCH}_{2} \mathrm{CH}=\mathrm{CH}_{\mathrm{a}} \underline{\mathrm{H}}_{\mathrm{b}}$ ), 6.02 (ddt, $\left.J=17.2,10.8,5.2 \mathrm{~Hz}, 1 \mathrm{H}, \mathrm{OCH}_{2} \mathrm{C} \underline{\mathrm{H}}=\mathrm{CH}_{2}\right), 6.84$ $(\mathrm{d}, J=8.8 \mathrm{~Hz}, 1 \mathrm{H} \mathrm{ArH}), 7.54(\mathrm{dd}, J=8.8,2.8 \mathrm{~Hz}, 1 \mathrm{H}, \mathrm{ArH}), 7.84(\mathrm{~d}, J=2.8 \mathrm{~Hz}, 1 \mathrm{H}, \operatorname{ArH})$, 
$10.38(\mathrm{~s}, 1 \mathrm{H}, \mathrm{CHO}) ;{ }^{13} \mathrm{C}-\mathrm{NMR}\left(\mathrm{CDCl}_{3}, 100 \mathrm{MHz}\right) \delta 69.3,113.4,114.8,118.4,126.1,130.7$, 131.8, 138.1, 159.6, 188.1; EI-MS (70 eV) $m / z$ (rel. intensity, \%) $242\left([\mathrm{M}+2]^{+}, 20\right), 240\left(\mathrm{M}^{+}\right.$, 21), 201 (48), 200 (44), 199 (56), 198 (34), 143 (24), 133 (70), 132 (100), 64 (27), 63 (54); HRMS calcd for $\mathrm{C}_{10} \mathrm{H}_{9} \mathrm{BrO}_{2}$ : 239.9786. Found: 239.9786.

2-Allyloxy-3,5-dichlorobenzaldehyde (2e). ${ }^{14}(21.85 \mathrm{~g}, 95 \%)$ was obtained as colorless crystal, mp $43-44^{\circ} \mathrm{C}, \mathrm{R}_{\mathrm{f}}=0.61$ (ethyl acetate: $n$-hexane $\left.=1: 15\right),{ }^{1} \mathrm{H}-\mathrm{NMR}\left(\mathrm{CDCl}_{3}, 400 \mathrm{MHz}\right) \delta 4.61(\mathrm{~d}$, $\left.J=6.4 \mathrm{~Hz}, 2 \mathrm{H}, \mathrm{OC}_{2} \mathrm{CH}=\mathrm{CH}_{2}\right), 5.32\left(\mathrm{~d}, J=10.4 \mathrm{~Hz}, 1 \mathrm{H}, \mathrm{OCH}_{2} \mathrm{CH}=\mathrm{CH}_{\mathrm{a}} \mathrm{H}_{\mathrm{b}}\right), 5.39(\mathrm{dd}, J=16.8$, $\left.1.2 \mathrm{~Hz}, 1 \mathrm{H}, \mathrm{OCH}_{2} \mathrm{CH}=\mathrm{CH}_{\mathrm{a}} \underline{\mathrm{H}}_{\mathrm{b}}\right), 6.07$ (ddt, $\left.J=16.8,10.4,6.4 \mathrm{~Hz}, 1 \mathrm{H}, \mathrm{OCH}_{2} \mathrm{C} \underline{\mathrm{H}}=\mathrm{CH}_{2}\right), 7.61(\mathrm{~d}, J$ $=2.8 \mathrm{~Hz}, 1 \mathrm{H}, \mathrm{ArH}), 7.69(\mathrm{~d}, J=2.8 \mathrm{~Hz}, 1 \mathrm{H}, \mathrm{ArH}), 10.27(\mathrm{~s}, 1 \mathrm{H}, \mathrm{CHO}) ;{ }^{13} \mathrm{C}-\mathrm{NMR}\left(\mathrm{CDCl}_{3}, 100\right.$ MHz) $\delta$ 76.6, 120.3, 126.5, 129.9, 130.4, 131.6, 131.7, 135.6, 156.2, 187.9; EI-MS (70eV) $\mathrm{m} / \mathrm{z}$ (rel. intensity, \%) 232 ([M+2] $\left.]^{+}, 5\right), 230\left(\mathrm{M}^{+}, 8\right), 203$ (44), 201 (69), 191 (59), 190 (72), 189 (100), 188 (95) , 167 (42), 135 (34), 133 (57), 97 (63); HRMS calcd for $\mathrm{C}_{10} \mathrm{H}_{8} \mathrm{Cl}_{2} \mathrm{O}_{2}$ : 229.9901. Found: 229.9902 .

\section{General procedure for the preparation of (2-allyloxyaryl)-2-propen-1-ol (3a-e)}

Under $\mathrm{N}_{2}$, the $O$-allyloxybenzaldehydes (2a-e) $(30 \mathrm{mmol})$ dissolved in anhydrous THF $(100 \mathrm{~mL})$ was stirred and cooled to $0^{\circ} \mathrm{C}$ and was subsequently added vinylmagnesium bromide $(1.6 \mathrm{M})$ $(22.5 \mathrm{~mL}, 36 \mathrm{mmol})$. The resulting mixture was stirred at $0^{\circ} \mathrm{C}$ for $0.5 \mathrm{hr}$ and then at room temperature for $2 \mathrm{hr}$ and then, quenched with saturated aq. $\mathrm{NH}_{4} \mathrm{Cl}$. The giving mixture was concentrated in vacuo to remove THF and the resulting residue was extracted with ethyl acetate $(20 \mathrm{~mL} \times 5)$. The organic layer was combined and washed with brine, and then dried over anhydrous $\mathrm{MgSO}_{4}$, and filtered in sequence. The giving filtrate was concentrated in vacuo to remove the solvent. The residue which was obtained was purified by silica gel column chromatography (ethyl acetate: $n$-hexane $=1: 15$ ) to give pure 3a-e.

1-(2-Allyloxyphenyl)-2-propen-1-ol (3a). $(4.60 \mathrm{~g}, 81 \%)$ was obtained as colorless liquid, $\mathrm{R}_{\mathrm{f}}=$ 0.38 (ethyl acetate: $n$-hexane $=1: 10),{ }^{1} \mathrm{H}-\mathrm{NMR}\left(\mathrm{CDCl}_{3}, 400 \mathrm{MHz}\right) \delta 2.87(\mathrm{br} \mathrm{d}, J=6.0 \mathrm{~Hz}, 1 \mathrm{H}$, $\mathrm{OH}), 4.57\left(\mathrm{dt}, J=5.2,1.2 \mathrm{~Hz}, 2 \mathrm{H}, \mathrm{OC}_{2} \mathrm{CH}=\mathrm{CH}_{2}\right.$ ), 5.16 (ddd, $J=10.4,1.2 \mathrm{~Hz}, 1.2 \mathrm{~Hz}, 1 \mathrm{H}$, $\left.\mathrm{CH}(\mathrm{OH})-\mathrm{CH}=\underline{\mathrm{CH}}_{\mathrm{a}} \mathrm{H}_{\mathrm{b}}\right), 5.29$ (ddt, $J=10.4,1.2,1.2 \mathrm{~Hz}, 1 \mathrm{H}, \mathrm{OCH}_{2} \mathrm{CH}=\mathrm{C}_{\mathrm{a}} \mathrm{H}_{\mathrm{b}}$ ), 5.32 (ddd, $J=$ 17.6, $1.2,1.2 \mathrm{~Hz}, 1 \mathrm{H}, \mathrm{CH}(\mathrm{OH}) \mathrm{CH}=\mathrm{CH}_{a} \underline{\mathrm{H}}_{\mathrm{b}}$ ), 5.41 (ddt, $J=17.6,1.2,1.2 \mathrm{~Hz}, 1 \mathrm{H}$, $\left.\mathrm{OCH}_{2} \mathrm{CH}=\mathrm{CH}_{a} \underline{\mathrm{H}}_{\mathrm{b}}\right), 5.44\left(\mathrm{~m}, 1 \mathrm{H}, \mathrm{C} \underline{\mathrm{H}}(\mathrm{OH}) \mathrm{CH}=\mathrm{CH}_{2}\right), 6.05$ (ddt, $J=17.6,10.4,5.2 \mathrm{~Hz}, 1 \mathrm{H}$, $\left.\mathrm{OCH}_{2} \mathrm{C} \underline{\mathrm{H}}=\mathrm{CH}_{2}\right), 6.13$ (ddd, $\left.J=17.6,10.4,5.6 \mathrm{~Hz}, 1 \mathrm{H}, \mathrm{CH}(\mathrm{OH}) \mathrm{C} \underline{\mathrm{H}}=\mathrm{CH}_{\mathrm{a}} \mathrm{H}_{\mathrm{b}}\right), 6.87(\mathrm{~m}, 1 \mathrm{H}, \mathrm{ArH})$, $6.96(\mathrm{~m}, 1 \mathrm{H}, \mathrm{ArH}), 7.23(\mathrm{~m}, 1 \mathrm{H}, \mathrm{ArH}), 7.31(\mathrm{~m}, 1 \mathrm{H}, \mathrm{ArH}) ;{ }^{13} \mathrm{C}-\mathrm{NMR}\left(\mathrm{CDCl}_{3}, 100 \mathrm{MHz}\right) \delta 68.9$, 71.6, 111.9, 114.5, 117.6, 121.1, 127.5, 128.6, 131.0, 132.0, 139.4, 155.7; EI-MS (70 eV) m/z (rel. intensity, \%) $190\left(\mathrm{M}^{+}, 6\right), 150$ (10), 149 (100), 147 (18), 133 (12), 132 (19), 131 (97), 121 (81), 107 (25), 103 (26), 93 (14), 91 (19); HRMS calcd for $\mathrm{C}_{12} \mathrm{H}_{14} \mathrm{O}_{2}$ : 190.0988. Found: 190.0986 .

1-(2-Allyloxy-4-methoxyphenyl)prop-2-en-1-ol (3b). (5.74 g, 87\%) was obtained as colorless liquid, $\mathrm{R}_{\mathrm{f}}=0.31$ (ethyl acetate: $n$-hexane $\left.=1: 9\right),{ }^{1} \mathrm{H}-\mathrm{NMR}\left(\mathrm{CDCl}_{3}, 200 \mathrm{MHz}\right) \delta 2.82(\mathrm{br} \mathrm{d}, J=$ $5.2 \mathrm{~Hz}, 1 \mathrm{H}, \mathrm{OH}), 3.77$ (s, $\left.3 \mathrm{H}, \mathrm{OCH}_{3}\right), 4.53\left(\mathrm{dt}, J=5.2,1.2 \mathrm{~Hz}, 2 \mathrm{H}, \mathrm{OCH}_{2} \mathrm{CH}=\mathrm{CH}_{2}\right), 5.14$ (ddd, $J$ $\left.=10.4,1.2,1.2 \mathrm{~Hz}, 1 \mathrm{H}, \mathrm{CH}(\mathrm{OH}) \mathrm{CH}=\mathrm{C}_{\mathrm{a}} \mathrm{H}_{\mathrm{b}}\right), 5.28(\mathrm{ddt}, J=10.4,1.4 \mathrm{~Hz}, 1.4 \mathrm{~Hz}, 1 \mathrm{H}$, 
$\left.\mathrm{OCH}_{2} \mathrm{CH}=\mathrm{CH}_{\mathrm{a}} \mathrm{H}_{\mathrm{b}}\right), 5.30\left(\mathrm{ddd}, J=17.2,1.2,1.2 \mathrm{~Hz}, 1 \mathrm{H}, \mathrm{CH}(\mathrm{OH}) \mathrm{CH}=\mathrm{CH}_{\mathrm{a}} \underline{\mathrm{H}}_{\mathrm{b}}\right), 5.40(\mathrm{~m}, 1 \mathrm{H}$, $\left.\mathrm{C} \underline{\mathrm{H}}(\mathrm{OH}) \mathrm{CH}=\mathrm{CH}_{2}\right), 5.41\left(\mathrm{ddt}, J=17.2,1.4 \mathrm{~Hz}, 1.4 \mathrm{~Hz}, 1 \mathrm{H}, \mathrm{OCH}_{2} \mathrm{CH}=\mathrm{CH}_{\mathrm{a}} \underline{\mathrm{H}}_{\mathrm{b}}\right), 6.04$ (ddt, $J=$ $\left.17.2, \quad 10.4,5.2 \mathrm{~Hz}, 1 \mathrm{H}, \quad \mathrm{OCH}_{2} \mathrm{C} \underline{\mathrm{H}}=\mathrm{CH}_{2}\right), 6.11$ (ddd, $J=17.2,10.4,5.4 \mathrm{~Hz}, 1 \mathrm{H}$, $\left.\mathrm{CH}(\mathrm{OH}) \mathrm{CH}=\mathrm{CH}_{2}\right), 6.44(\mathrm{~d}, J=2.4 \mathrm{~Hz}, 1 \mathrm{H}, \mathrm{ArH}), 6.47(\mathrm{dd}, J=8.0,2.4 \mathrm{~Hz}, 1 \mathrm{H}, \mathrm{ArH}), 7.20(\mathrm{~d}, J$ $=8.0 \mathrm{~Hz}, 1 \mathrm{H}, \mathrm{ArH}) ;{ }^{13} \mathrm{C}-\mathrm{NMR}\left(\mathrm{CDCl}_{3}, 50 \mathrm{MHz}\right) \delta 55.2,68.8,70.8,99.8,104.5,114.1,117.6$, 123.7, 128.0, 132.8, 139.7, 156.6, 160.1; EI-MS (70 eV) $\mathrm{m} / z$ (rel. intensity, \%) $221\left([\mathrm{M}+1]^{+}, 4\right)$, $220\left(\mathrm{M}^{+}, 21\right), 203$ (100), 202 (50), 201 (13), 164 (15), 161 (13), 151 (11); HRMS calcd for $\mathrm{C}_{13} \mathrm{H}_{16} \mathrm{O}_{3}: 220.1099$. Found: 220.1099.

1-(2-Allyloxy-3-methoxyphenyl)prop-2-en-1-ol (3c). ${ }^{15}$ (5.62 g, 85\%) was obtained as colorless liquid, $\mathrm{R}_{\mathrm{f}}=0.33$ (ethyl acetate: $n$-hexane $\left.=1: 9\right),{ }^{1} \mathrm{H}$-NMR $\left(\mathrm{CDCl}_{3}, 400 \mathrm{MHz}\right) \delta 2.93(\mathrm{br} \mathrm{d}, J=$ $4.4 \mathrm{~Hz}, 1 \mathrm{H}, \mathrm{OH}), 3.83\left(\mathrm{~s}, 3 \mathrm{H}, \mathrm{OCH}_{3}\right), 4.52\left(\mathrm{ddt}, J=12.0,5.6,1.6 \mathrm{~Hz}, 1 \mathrm{H}, \mathrm{OC}_{\mathrm{a}} \mathrm{H}_{\mathrm{b}} \mathrm{CH}=\mathrm{CH}_{2}\right)$, 4.56 (ddt, $J=12.0,5.6,1.6 \mathrm{~Hz}, 1 \mathrm{H}, \mathrm{OCH}_{\mathrm{a}} \underline{\mathrm{H}}_{\mathrm{b}} \mathrm{CH}=\mathrm{CH}_{2}$ ), 5.15 (ddd, $J=10.4,1.6,1.6 \mathrm{~Hz}, 1 \mathrm{H}$, $\left.\mathrm{CH}(\mathrm{OH}) \mathrm{CH}=\underline{\mathrm{CH}}_{\mathrm{a}} \mathrm{H}_{\mathrm{b}}\right), 5.23\left(\mathrm{ddt}, J=10.4,1.6,1.6 \mathrm{~Hz}, 1 \mathrm{H}, \mathrm{OCH}_{2} \mathrm{CH}=\mathrm{C}_{\mathrm{H}} \mathrm{H}_{\mathrm{b}}\right), 5.31$ (ddd, $J=$ $\left.17.2,1.6,1.6 \mathrm{~Hz}, 1 \mathrm{H}, \mathrm{CH}(\mathrm{OH}) \mathrm{CH}=\mathrm{CH}_{\mathrm{a}} \underline{\mathrm{H}}_{\mathrm{b}}\right), 5.36\left(\mathrm{ddt}, J=17.2,1.6,1.6 \mathrm{~Hz}, 1 \mathrm{H}, \mathrm{OCH}_{2} \mathrm{CH}=\right.$ $\left.\mathrm{CH}_{\mathrm{a}} \underline{\mathrm{H}}_{\mathrm{b}}\right), 5.47\left(\mathrm{~m}, 1 \mathrm{H}, \mathrm{C} \underline{\mathrm{H}}(\mathrm{OH}) \mathrm{CH}=\mathrm{CH}_{2}\right), 6.07$ (ddd, $J=17.2,10.4,5.2 \mathrm{~Hz}, 1 \mathrm{H}, \mathrm{CH}(\mathrm{OH}) \mathrm{C} \underline{\mathrm{H}}=$ $\mathrm{CH}_{2}$ ), 6.07 (ddt, $\left.J=17.2,10.4,5.6 \mathrm{~Hz}, 1 \mathrm{H}, \mathrm{OCH}_{2} \mathrm{CH}=\mathrm{CH}_{2}\right), 6.84(\mathrm{dd}, J=8.0,1.6 \mathrm{~Hz}, 1 \mathrm{H}, \mathrm{ArH})$, $6.93(\mathrm{dd}, J=8.0,1.6 \mathrm{~Hz}, 1 \mathrm{H}, \mathrm{ArH}), 7.04(\mathrm{t}, J=8.0 \mathrm{~Hz}, 1 \mathrm{H}, \mathrm{ArH}) ;{ }^{13} \mathrm{C}-\mathrm{NMR}\left(\mathrm{CDCl}_{3}, 100\right.$ $\mathrm{MHz}) \delta 55.6,70.6,73.7,111.6,114.3,117.5,119.1,124.1,134.0,136.4,139.9,146.0,152.4$; EIMS (70 eV) m/z (rel. intensity, \%) $220\left(\mathrm{M}^{+}, 19\right), 204$ (17), 203 (100), 202 (59), 201 (11), 164 (23), 163 (11), 162 (20), 161 (32); HRMS calcd for $\mathrm{C}_{13} \mathrm{H}_{16} \mathrm{O}_{3}: 220.1099$. Found: 220.1100.

1-(2-Allyloxy-5-bromophenyl)prop-2-en-1-ol (3d). (5.85 g, 73\%) was obtained as colorless liquid, $\mathrm{R}_{\mathrm{f}}=0.32$ (ethyl acetate: $n$-hexane $\left.=1: 9\right),{ }^{1} \mathrm{H}$-NMR $\left(\mathrm{CDCl}_{3}, 200 \mathrm{MHz}\right) \delta 2.73($ br s, $1 \mathrm{H}$, $\mathrm{OH}), 4.54\left(\mathrm{dt}, J=5.2,1.6 \mathrm{~Hz}, 2 \mathrm{H}, \mathrm{OCH}_{2} \mathrm{CH}=\mathrm{CH}_{2}\right), 5.18(\mathrm{ddd}, J=10.4,1.6,1.6 \mathrm{~Hz}, 1 \mathrm{H}$, $\left.\mathrm{CH}(\mathrm{OH}) \mathrm{CH}=\underline{\mathrm{CH}}_{\mathrm{a}} \mathrm{H}_{\mathrm{b}}\right), 5.30$ (ddt, $\left.J=10.4,1.6,1.6 \mathrm{~Hz}, 1 \mathrm{H}, \mathrm{OCH}_{2} \mathrm{CH}=\mathrm{C}_{\mathrm{H}} \mathrm{H}_{\mathrm{b}}\right), 5.33$ (ddd, $J=$ $\left.17.2,1.6,1.6 \mathrm{~Hz}, 1 \mathrm{H}, \mathrm{CH}(\mathrm{OH}) \mathrm{CH}=\mathrm{CH}_{\mathrm{a}} \underline{\mathrm{H}}_{\mathrm{b}}\right), 5.39$ (ddt, $J=17.2,1.6,1.6 \mathrm{~Hz}, 1 \mathrm{H}, \mathrm{OCH}_{2} \mathrm{CH}=$ $\left.\mathrm{CH}_{\mathrm{a}} \underline{\mathrm{H}}_{\mathrm{b}}\right), 5.42\left(\mathrm{~m}, 1 \mathrm{H}, \mathrm{C} \underline{\mathrm{H}}(\mathrm{OH}) \mathrm{CH}=\mathrm{CH}_{2}\right), 6.02\left(\mathrm{ddt}, J=17.2,10.4,5.2 \mathrm{~Hz}, 1 \mathrm{H}, \mathrm{OCH}_{2} \mathrm{CH}=\mathrm{CH}_{2}\right)$, $6.06\left(\mathrm{ddd}, J=17.2,10.4,5.4 \mathrm{~Hz}, 1 \mathrm{H}, \mathrm{CH}(\mathrm{OH}) \mathrm{C} \underline{\mathrm{H}}=\mathrm{CH}_{2}\right), 6.73(\mathrm{~d}, J=8.8 \mathrm{~Hz}, 1 \mathrm{H}, \mathrm{ArH}), 7.32$ $(\mathrm{dd}, J=8.8,2.6 \mathrm{~Hz}, 1 \mathrm{H}, \mathrm{ArH}), 7.46(\mathrm{~d}, J=2.6 \mathrm{~Hz}, 1 \mathrm{H}, \mathrm{ArH}) ;{ }^{13} \mathrm{C}-\mathrm{NMR}\left(\mathrm{CDCl}_{3}, 50 \mathrm{MHz}\right) \delta$ 69.1, 70.5, 113.4, 113.6, 115.1, 117.9, 130.1, 131.1, 132.58, 133.3, 138.7, 154.6; EI-MS (70 eV) $m / z$ (rel. intensity, \%) $270\left([\mathrm{M}+2]^{+}, 17\right), 268\left(\mathrm{M}^{+}, 17\right), 211$ (71), 209 (72), 148 (57), 132 (41), 131 (100), 120 (72), 103 (62), 91 (56); HRMS calcd for $\mathrm{C}_{12} \mathrm{H}_{13} \mathrm{BrO}_{2}$ : 268.0099. Found: 268.1100 .

1-(2-Allyloxy-3,5-dichlorophenyl)prop-2-en-1-ol (3e). (5.81 g, 75\%) was obtained as colorless liquid, $\mathrm{R}_{\mathrm{f}}=0.35$ (ethyl acetate: $n$-hexane $\left.=1: 9\right),{ }^{1} \mathrm{H}-\mathrm{NMR}\left(\mathrm{CDCl}_{3}, 200 \mathrm{MHz}\right) \delta 2.39(\mathrm{~d}, J=4.6$ $\mathrm{Hz}, 1 \mathrm{H}, \mathrm{OH}), 4.52\left(\mathrm{dt}, J=5.6,1.6 \mathrm{~Hz}, 2 \mathrm{H}, \mathrm{OC}_{2} \mathrm{CH}=\mathrm{CH}_{2}\right), 5.24$ (ddd, $J=10.2,1.4,1.4 \mathrm{~Hz}, 1 \mathrm{H}$, $\left.\mathrm{CH}(\mathrm{OH}) \mathrm{CH}=\underline{\mathrm{CH}}_{\mathrm{a}} \mathrm{H}_{\mathrm{b}}\right), 5.30\left(\mathrm{ddt}, J=10.2,1.6,1.6 \mathrm{~Hz}, 1 \mathrm{H}, \mathrm{OCH}_{2} \mathrm{CH}=\mathrm{CH}_{\mathrm{a}} \mathrm{H}_{\mathrm{b}}\right), 5.37$ (ddd, $J=$ $\left.17.2,1.4,1.4 \mathrm{~Hz}, 1 \mathrm{H}, \mathrm{CH}(\mathrm{OH}) \mathrm{CH}=\mathrm{CH}_{\mathrm{a}} \underline{\mathrm{H}}_{\mathrm{b}}\right), 5.42$ (ddt, $J=17.0,1.6,1.6 \mathrm{~Hz}, 1 \mathrm{H}, \mathrm{OCH}_{2} \mathrm{CH}=$ $\left.\mathrm{CH}_{\mathrm{a}} \underline{\mathrm{H}}_{\mathrm{b}}\right), 5.50\left(\mathrm{~m}, 1 \mathrm{H}, \mathrm{C} \underline{\mathrm{H}}(\mathrm{OH}) \mathrm{CH}=\mathrm{CH}_{2}\right), 6.02(\mathrm{ddd}, J=17.2,10.2,5.4 \mathrm{~Hz}, 1 \mathrm{H}, \mathrm{CH}(\mathrm{OH}) \mathrm{C} \underline{H}=$ $\mathrm{CH}_{2}$ ), 6.10 (ddt, $\left.J=17.0,10.2,5.4 \mathrm{~Hz}, 1 \mathrm{H}, \mathrm{OCH}_{2} \mathrm{CH}=\mathrm{CH}_{2}\right), 7.30$ (d, $\left.J=2.6 \mathrm{~Hz}, 1 \mathrm{H}, \mathrm{ArH}\right), 7.32$ $(\mathrm{d}, J=2.6 \mathrm{~Hz}, 1 \mathrm{H}, \mathrm{ArH}) ;{ }^{13} \mathrm{C}-\mathrm{NMR}\left(\mathrm{CDCl}_{3}, 50 \mathrm{MHz}\right) \delta 69.7,74.6,115.8,118.6,126.3,128.6$, 
129.4 129.9, 132.8, 138.8, 139.1, 150.9; EI-MS (70 eV) m/z (rel. intensity, \%) $260\left([\mathrm{M}+2]^{+}\right.$, 53\%), $258\left(\mathrm{M}^{+}, 1\right), 203$ (16), 202 (42), 201 (71), 200 (66), 199 (100), 189 (17) , 167 (19), 165 (39), 137 (18); HRMS calcd for $\mathrm{C}_{12} \mathrm{H}_{12} \mathrm{Cl}_{2} \mathrm{O}_{2}$ : 258.0214. Found: 258.0214 .

\section{General procedure for the preparation of (2-allyloxyphenyl)-2-propen-1-one (4a-e)}

The (2-allyloxyphenyl)-2-propen-1-ol (3a-e) $(20 \mathrm{mmol})$ which was dissolved in anhydrous $\mathrm{CH}_{2} \mathrm{Cl}_{2}(85 \mathrm{~mL})$ was added $\mathrm{MnO}_{2}(200 \mathrm{mmol})$. The mixture was stirred at room temperature for $5 \mathrm{hr}$. After concentration in vacuo, the residue which was obtained was purified by silica gel column chromatography (ethyl acetate: $n$-hexane $=1: 20$ ) to give pure 4a-e.

1-(2-Allyloxyphenyl)-2-propen-1-one (4a). (3.01 g, 80\%) was obtained as colorless liquid, $\mathrm{R}_{\mathrm{f}}=$ 0.53 (ethyl acetate: $n$-hexane $=1: 10),{ }^{1} \mathrm{H}-\mathrm{NMR}\left(\mathrm{CDCl}_{3}, 400 \mathrm{MHz}\right) \delta 4.61(\mathrm{dt}, J=5.2,1.2 \mathrm{~Hz}$, $2 \mathrm{H}, \mathrm{OC}_{2} \mathrm{CH}=\mathrm{CH}_{2}$ ), 5.28 (ddt, $J=10.4,1.2,1.2 \mathrm{~Hz}, 1 \mathrm{H}, \mathrm{OCH}_{2} \mathrm{CH}=\mathrm{CH}_{2}$ ), 5.42 (ddt, $J=17.2$, $\left.1.2,1.2 \mathrm{~Hz}, 1 \mathrm{H}, \mathrm{OCH}_{2} \mathrm{CH}=\underline{\mathrm{C}}_{2}\right), 5.79\left(\mathrm{dd}, J=10.4,1.6 \mathrm{~Hz}, 1 \mathrm{H}, \mathrm{COCH}=\underline{\mathrm{C}}_{\mathrm{a}} \mathrm{H}_{\mathrm{b}}\right), 6.03$ (ddt, $J=$ 17.2, 10.4, $\left.5.2 \mathrm{~Hz}, 1 \mathrm{H}, \mathrm{OCH}_{2} \mathrm{C} \underline{\mathrm{H}}=\mathrm{CH}_{2}\right), 6.28\left(\mathrm{dd}, J=17.2 \mathrm{~Hz}, 1.6 \mathrm{~Hz}, 1 \mathrm{H}, \mathrm{COCH}=\mathrm{CH}_{a} \underline{H}_{\mathrm{b}}\right), 6.95$ (m, 1H, ArH), $7.00(\mathrm{~m}, 1 \mathrm{H}, \mathrm{ArH}), 7.04\left(\mathrm{dd}, J=17.2,10.4 \mathrm{~Hz}, 1 \mathrm{H}, \mathrm{COCH}=\mathrm{C}_{2}\right), 7.43(\mathrm{~m}, 1 \mathrm{H}$, $\mathrm{ArH}), 7.58(\mathrm{~m}, 1 \mathrm{H}, \mathrm{ArH}) ;{ }^{13} \mathrm{C}-\mathrm{NMR}\left(\mathrm{CDCl}_{3}, 100 \mathrm{MHz}\right) \delta 69.3,112.9,117.6,120.9,128.2$, 128.9, 130.5, 132.5, 132.9, 136.7, 157.2, 193.3; EI-MS (70 eV) m/z (rel. intensity, \%) 189 $\left([\mathrm{M}+1]^{+}, 6\right), 188\left(\mathrm{M}^{+}, 2\right), 170$ (16), 169 (26), 147 (38), 131 (24), 121 (100), 91 (22); HRMS calcd for $\mathrm{C}_{12} \mathrm{H}_{12} \mathrm{O}_{2}: 188.0837$. Found: 188.0839 .

1-(2-allyloxy-4-methoxyphenyl)-2-propen-1-one (4b). (3.08 g, 70\%) was obtained as colorless liquid, $\mathrm{R}_{\mathrm{f}}=0.38$ (ethyl acetate: $n$-hexane $\left.=1: 9\right),{ }^{1} \mathrm{H}-\mathrm{NMR}\left(\mathrm{CDCl}_{3}, 200 \mathrm{MHz}\right) \delta 3.83(\mathrm{~s}, 3 \mathrm{H}$, $\left.\mathrm{OCH}_{3}\right), 4.59\left(\mathrm{dt}, J=5.2,1.6 \mathrm{~Hz}, 2 \mathrm{H}, \mathrm{OCH}_{2} \mathrm{CH}=\mathrm{CH}_{2}\right), 5.30(\mathrm{ddt}, J=10.6,1.6,1.6 \mathrm{~Hz}, 1 \mathrm{H}$, $\mathrm{OCH}_{2} \mathrm{CH}=\mathrm{C}_{\mathrm{H}} \mathrm{H}_{\mathrm{b}}$ ), 5.44 (ddt, $\left.J=17.2,1.6,1.6 \mathrm{~Hz}, 1 \mathrm{H}, \mathrm{OCH}_{2} \mathrm{CH}=\mathrm{CH}_{\mathrm{a}} \underline{\mathrm{H}}_{\mathrm{b}}\right), 5.70$ (dd, $J=10.4$, $\left.1.8 \mathrm{~Hz}, 1 \mathrm{H}, \mathrm{COCH}=\mathrm{CH}_{\mathrm{a}} \mathrm{H}_{\mathrm{b}}\right), 6.04\left(\mathrm{ddt}, J=17.2,10.6,5.2 \mathrm{~Hz}, 1 \mathrm{H}, \mathrm{OCH}_{2} \mathrm{C} \underline{\mathrm{H}}=\mathrm{CH}_{2}\right), 6.32(\mathrm{dd}, J=$ $\left.17.2,1.8 \mathrm{~Hz}, 1 \mathrm{H}, \mathrm{COCH}=\mathrm{CH}_{\mathrm{a}} \underline{\mathrm{H}}_{\mathrm{b}}\right), 6.45(\mathrm{~d}, J=2.2 \mathrm{~Hz}, 1 \mathrm{H}, \mathrm{ArH}), 6.54(\mathrm{dd}, J=8.8,2.2 \mathrm{~Hz}, 1 \mathrm{H}$, ArH), $7.18\left(\mathrm{dd}, J=17.2,10.4 \mathrm{~Hz}, 1 \mathrm{H}, \mathrm{COC} \underline{\mathrm{H}}=\mathrm{CH}_{2}\right), 7.71(\mathrm{~d}, J=8.8 \mathrm{~Hz}, 1 \mathrm{H}, \mathrm{ArH}) ;{ }^{13} \mathrm{C}-\mathrm{NMR}$ $\left(\mathrm{CDCl}_{3}, 50 \mathrm{MHz}\right) \delta 55.4,69.3,99.6,105.7,117.7,121.7,126.7,132.3,132.8,136.8,159.4$, 164.1, 190.7; EI-MS (70eV) m/z (rel. intensity, \%) 219 ([M+1] $\left.]^{+}, 68\right), 218\left(\mathrm{M}^{+}, 48\right), 217$ (29), 188 (32), 163 (40), 151 (100), 121 (42), 91 (42), 77 (38), 63 (38); HRMS calcd for $\mathrm{C}_{13} \mathrm{H}_{14} \mathrm{O}_{3}$ : 218.0943. Found: 218.0945.

1-(2-Allyloxy-3-methoxyphenyl)-2-propen-1-one $\quad(\mathbf{4 c}) .^{15}$ (3.24 g, 74\%) was obtained as colorless liquid, $\mathrm{R}_{\mathrm{f}}=0.38$ (ethyl acetate: $n$-hexane $\left.=1: 9\right),{ }^{1} \mathrm{H}-\mathrm{NMR}\left(\mathrm{CDCl}_{3}, 200 \mathrm{MHz}\right) \delta 3.87$ $\left(\mathrm{s}, 3 \mathrm{H}, \mathrm{OCH}_{3}\right), 4.50\left(\mathrm{dt}, J=5.8,1.2 \mathrm{~Hz}, 2 \mathrm{H}, \mathrm{OC}_{2} \mathrm{CH}=\mathrm{CH}_{2}\right.$ ), 5.17 (ddt, $J=10.2,1.2,1.2 \mathrm{~Hz}$, $\left.1 \mathrm{H}, \mathrm{OCH}_{2} \mathrm{CH}=\mathrm{C}_{\mathrm{H}_{\mathrm{a}}} \mathrm{H}_{\mathrm{b}}\right), 5.28\left(\mathrm{ddt}, J=17.2,1.2,1.2 \mathrm{~Hz}, 1 \mathrm{H}, \mathrm{OCH}_{2} \mathrm{CH}=\mathrm{CH}_{\mathrm{a}} \underline{\mathrm{H}}_{\mathrm{b}}\right), 5.86(\mathrm{dd}, J=$ $\left.10.4,1.4 \mathrm{~Hz}, 1 \mathrm{H}, \mathrm{COCH}=\mathrm{C}_{\mathrm{H}} \mathrm{H}_{\mathrm{b}}\right), 6.00\left(\mathrm{ddt}, J=17.2,10.2,5.8 \mathrm{~Hz}, 1 \mathrm{H}, \mathrm{OCH}_{2} \mathrm{C} \underline{\mathrm{H}}=\mathrm{CH}_{2}\right), 6.22$ $\left(\mathrm{dd}, J=17.6,1.4 \mathrm{~Hz}, 1 \mathrm{H}, \mathrm{COCH}=\mathrm{CH}_{a} \underline{H}_{\mathrm{b}}\right), 6.94\left(\mathrm{dd}, J=17.2,10.4 \mathrm{~Hz}, 1 \mathrm{H}, \mathrm{COCH}=\mathrm{CH}_{2}\right) 7.07$ $(\mathrm{m}, 3 \mathrm{H}, \mathrm{ArH}) ;{ }^{13} \mathrm{C}-\mathrm{NMR}\left(\mathrm{CDCl}_{3}, 50 \mathrm{MHz}\right) \delta 55.8,74.8,115.0,117.7,120.7,123.9,129.2$, 133.4, 133.9, 136.5, 146.3, 152.7, 193.6; EI-MS (70eV) $\mathrm{m} / z$ (rel. intensity, \%) 219 ([M+1] $]^{+}$, 100), 218 ( $\left.\mathrm{M}^{+}, 34\right), 177$ (22), 164 (13), 162 (15), 151 (52), 150 (20), 122 (21), 121 (35), 91 (22); HRMS calcd for $\mathrm{C}_{13} \mathrm{H}_{14} \mathrm{O}_{3}: 218.0943$. Found: 218.0943 . 
1-(2-Allyloxy-5-bromophenyl)-2-propen-1-one (4d). (3.79 g, 71\%) was obtained as colorless liquid, $\mathrm{R}_{\mathrm{f}}=0.50$ (ethyl acetate: $n$-hexane $\left.=1: 9\right),{ }^{1} \mathrm{H}-\mathrm{NMR}\left(\mathrm{CDCl}_{3}, 200 \mathrm{MHz}\right) \delta 4.59(\mathrm{dt}, J=5.2$, $\left.1.4 \mathrm{~Hz}, 2 \mathrm{H}, \mathrm{OCH}_{2} \mathrm{CH}=\mathrm{CH}_{2}\right), 5.29\left(\mathrm{ddt}, J=10.6,1.4,1.4 \mathrm{~Hz}, 1 \mathrm{H}, \mathrm{OCH}_{2} \mathrm{CH}=\mathrm{C}_{\mathrm{a}} \mathrm{H}_{\mathrm{b}}\right), 5.40(\mathrm{ddt}, J$ $\left.=17.2,1.4,1.4 \mathrm{~Hz}, 1 \mathrm{H}, \mathrm{OCH}_{2} \mathrm{CH}=\mathrm{CH}_{\mathrm{a}} \underline{\mathrm{H}}_{\mathrm{b}}\right), 5.84\left(\mathrm{dd}, J=10.2,1.6 \mathrm{~Hz}, 1 \mathrm{H}, \mathrm{COCH}=\underline{\mathrm{CH}}_{\mathrm{a}} \mathrm{H}_{\mathrm{b}}\right), 6.00$ (ddt, $\left.J=17.2,10.6,5.2 \mathrm{~Hz}, 1 \mathrm{H}, \mathrm{OCH}_{2} \mathrm{CH}=\mathrm{CH}_{2}\right), 6.29(\mathrm{dd}, J=17.2,1.6 \mathrm{~Hz}, 1 \mathrm{H}$, $\left.\mathrm{COCH}=\mathrm{CH}_{\mathrm{a}} \underline{\mathrm{H}}_{\mathrm{b}}\right), 6.84(\mathrm{~d}, J=8.8 \mathrm{~Hz}, 1 \mathrm{H}, \mathrm{ArH}), 6.99\left(\mathrm{dd}, J=17.2,10.2 \mathrm{~Hz}, 1 \mathrm{H}, \mathrm{COCH}=\mathrm{CH}_{2}\right)$, $7.51(\mathrm{dd}, J=8.8,2.6 \mathrm{~Hz}, 1 \mathrm{H}, \mathrm{ArH}), 7.67$ (d, $J=2.6 \mathrm{~Hz}, 1 \mathrm{H}, \mathrm{ArH}) ;{ }^{13} \mathrm{C}-\mathrm{NMR}\left(\mathrm{CDCl}_{3}, 50\right.$ $\mathrm{MHz}) \delta$ 69.6, 113.3, 114.8, 118.0, 129.0, 130.4, 132.1, 132.9, 135.3, 136.2, 156.2, 191.6; EI-MS $(70 \mathrm{eV}) \mathrm{m} / z$ (rel. intensity, \%) $268\left([\mathrm{M}+2]^{+}, 5\right), 267\left([\mathrm{M}+1]^{+}, 10\right), 266\left(\mathrm{M}^{+}, 5\right), 265\left([\mathrm{M}-1]^{+}, 9\right)$, 227 (33), 225 (36), 201 (96), 199 (100), 198 (67), 170 (24) , 169 (31), 131 (24), 119 (25), 90 (35), 89 (31), 63 (62); HRMS calcd for $\mathrm{C}_{12} \mathrm{H}_{11} \mathrm{BrO}_{2}$ : 265.9942. Found: 265.9944.

1-(2-Allyloxy-3,5-dichlorophenyl)-2-propen-1-one (4e). (3.75 g, 73\%) was obtained as colorless liquid, $\mathrm{R}_{\mathrm{f}}=0.61$ (ethyl acetate: $n$-hexane $\left.=1: 15\right),{ }^{1} \mathrm{H}-\mathrm{NMR}\left(\mathrm{CDCl}_{3}, 400 \mathrm{MHz}\right) \delta 4.43$ $\left(\mathrm{dt}, J=6.0,1.2 \mathrm{~Hz}, 2 \mathrm{H}, \mathrm{OC}_{2}{ }_{2} \mathrm{CH}=\mathrm{CH}_{2}\right), 5.25$ (ddt, $J=10.4,1.2,1.2 \mathrm{~Hz}, 1 \mathrm{H}, \mathrm{OCH}_{2} \mathrm{CH}=\mathrm{CH}_{\mathrm{a}} \mathrm{H}_{\mathrm{b}}$ ), 5.32 (ddt, $J=17.2,1.2,1.2 \mathrm{~Hz}, 1 \mathrm{H}, \mathrm{OCH}_{2} \mathrm{CH}=\mathrm{CH}_{\mathrm{a}} \underline{\mathrm{H}}_{\mathrm{b}}$ ), $5.98(\mathrm{dd}, J=10.4,1.2 \mathrm{~Hz}, 1 \mathrm{H}$, $\left.\mathrm{COCH}=\underline{\mathrm{CH}}_{\mathrm{a}} \mathrm{H}_{\mathrm{b}}\right), 5.99\left(\mathrm{ddt}, J=17.2,10.4,6.0 \mathrm{~Hz}, 1 \mathrm{H}, \mathrm{OCH}_{2} \mathrm{CH}=\mathrm{CH}_{2}\right), 6.29(\mathrm{dd}, J=17.6,1.2$ $\left.\mathrm{Hz}, 1 \mathrm{H}, \mathrm{COCH}=\mathrm{CH}_{\mathrm{a}} \underline{\mathrm{H}}_{\mathrm{b}}\right), 6.91\left(\mathrm{dd}, J=17.6,10.4 \mathrm{~Hz}, 1 \mathrm{H}, \mathrm{COC} \underline{\mathrm{H}}=\mathrm{CH}_{2}\right), 7.38(\mathrm{~d}, J=2.8 \mathrm{~Hz}, 1 \mathrm{H}$, $\operatorname{ArH}), 7.52(\mathrm{~d}, J=2.8 \mathrm{~Hz}, 1 \mathrm{H}, \mathrm{ArH}) ;{ }^{13} \mathrm{C}-\mathrm{NMR}\left(\mathrm{CDCl}_{3}, 100 \mathrm{MHz}\right) \delta 76.3,119.4,128.1,129.8$, 129.9, 131.4, 132.3, 132.8, 135.5, 135.7, 152.0, 191.5; EI-MS (70 eV) $\mathrm{m} / \mathrm{z}$ (rel. intensity, \%) 258 $\left([\mathrm{M}+2]^{+}, 1\right), 257\left([\mathrm{M}+1]^{+}, 6\right), 256\left(\mathrm{M}^{+}, 2\right), 255\left([\mathrm{M}-1]^{+}, 7\right), 217(25), 215(42), 202(24), 191$ (64), 190 (64), 189 (100) , 188 (81), 161 (23), 159 (28), 133 (21), 97 (33); HRMS calcd for $\mathrm{C}_{12} \mathrm{H}_{10} \mathrm{Cl}_{2} \mathrm{O}_{2}$ : 256.0058. Found: 256.0060 .

\section{General procedure for the preparation of $2 \mathrm{H}$-1-benzoxepin-5-one (5a-e)}

The (2-allyloxyphenyl)-2-propen-1-one (4a-e) (10 mmol) dissolved in anhydrous $\mathrm{CH}_{2} \mathrm{Cl}_{2}(100$ $\mathrm{mL}$ ) was added Grubbs' Catalyst $(5 \mathrm{~mol} \%$ ) and the mixture was stirred at room temperature for $6 \mathrm{hr}$. Then, the mixture was concentrated in vacuo to remove the solvent. The resulting residue was purified by silica gel column chromatography (ethyl acetate: $n$-hexane $=1: 20$ ) to give pure 5a-e.

2H-1-Benzoxepin-5-one (5a). ${ }^{4}(1.3 \mathrm{~g}, 81 \%)$ was obtained as colorless liquid, $\mathrm{R}_{\mathrm{f}}=0.35$ (ethyl acetate: $n$-hexane $=1: 10),{ }^{1} \mathrm{H}-\mathrm{NMR}\left(\mathrm{CDCl}_{3}, 400 \mathrm{MHz}\right) \delta 4.55\left(\mathrm{dd}, J=4.8,1.2 \mathrm{~Hz}, 2 \mathrm{H}, \mathrm{OC}_{2^{-}}\right.$ $\mathrm{CH}=\mathrm{CHCO}), 6.43\left(\mathrm{dt}, J=11.6,1.2 \mathrm{~Hz}, 1 \mathrm{H}, \mathrm{OCH}_{2} \mathrm{CH}=\mathrm{C} \underline{\mathrm{HCO}}\right), 6.75(\mathrm{dt}, J=11.6,4.8 \mathrm{~Hz}, 1 \mathrm{H}$, $\left.\mathrm{OCH}_{2} \mathrm{C} \underline{\mathrm{H}}=\mathrm{CHCO}\right), 7.09(\mathrm{~m}, 1 \mathrm{H}, \mathrm{ArH}), 7.17(\mathrm{~m}, 1 \mathrm{H}, \mathrm{ArH}), 7.47(\mathrm{~m}, 1 \mathrm{H}, \mathrm{ArH}), 7.95(\mathrm{~m}, 1 \mathrm{H}$, $\mathrm{ArH}) ;{ }^{13} \mathrm{C}-\mathrm{NMR}\left(\mathrm{CDCl}_{3}, 100 \mathrm{MHz}\right) \delta$ 68.8, 121.4, 123.9, 129.9, 131.2, 134.4, 134.8, 141.6, 159.0, 189.9; EI-MS (70 eV) m/z (rel. intensity, \%) $161\left([\mathrm{M}+1]^{+}, 11\right), 160\left(\mathrm{M}^{+}, 100\right), 132$ (37), 131 (98), 104 (15), 103 (21), 77 (12); HRMS calcd for $\mathrm{C}_{10} \mathrm{H}_{8} \mathrm{O}_{2}: 160.0519$. Found: 160.0521.

8-Methoxy-2H-1-benzoxepin-5-one (5b). (1.45 g, 76\%) was obtained as colorless liquid, $\mathrm{R}_{\mathrm{f}}=$ 0.26 (ethyl acetate: $n$-hexane $=1: 9),{ }^{1} \mathrm{H}-\mathrm{NMR}\left(\mathrm{CDCl}_{3}, 200 \mathrm{MHz}\right) \delta 3.83\left(\mathrm{~s}, 3 \mathrm{H}, \mathrm{OCH}_{3}\right), 4.68$ $\left(\mathrm{dd}, J=5.2,1.2 \mathrm{~Hz}, 2 \mathrm{H}, \mathrm{OC}_{2} \mathrm{CH}=\mathrm{CHCO}\right), 6.41\left(\mathrm{dt}, J=11.6,1.2 \mathrm{~Hz}, 1 \mathrm{H}, \mathrm{OCH}_{2} \mathrm{CH}=\mathrm{C} \underline{\mathrm{HCO}}\right)$, $6.52(\mathrm{~d}, J=2.4 \mathrm{~Hz}, 1 \mathrm{H}, \mathrm{ArH}), 6.69\left(\mathrm{dt}, J=11.6,5.2 \mathrm{~Hz}, 1 \mathrm{H}, \mathrm{OCH}_{2} \mathrm{C} \underline{\mathrm{H}}=\mathrm{CHCO}\right), 6.70(\mathrm{dd}, J=$ 
9.0, 2.4 Hz, 1H, ArH), 7.97 (d, $J=9.0 \mathrm{~Hz}, 1 \mathrm{H}, \mathrm{ArH}) ;{ }^{13} \mathrm{C}-\mathrm{NMR}\left(\mathrm{CDCl}_{3}, 50 \mathrm{MHz}\right) \delta 55.6,68.3$, 104.7, 111.0, 122.2, 133.2, 135.6, 139.4, 161.4, 164.98, 188.1; EI-MS (70 eV) m/z (rel. intensity, \%) $191\left([\mathrm{M}+1]^{+}, 34\right), 190\left(\mathrm{M}^{+}, 91\right), 62$ (75), 161 (100), 147 (25), 106 (20), 91 (24), 63 (62), 62 (21), 51 (27); HRMS calcd for $\mathrm{C}_{11} \mathrm{H}_{10} \mathrm{O}_{3}$ : 190.0630. Found: 190.0630 .

9-Methoxy-2H-1-benzoxepin-5-one (5c). (1.58 g, 83\%) was obtained as colorless crystal, mp $81-82^{\circ} \mathrm{C}, \mathrm{R}_{\mathrm{f}}=0.18$ (ethyl acetate: $n$-hexane $\left.=1: 10\right),{ }^{1} \mathrm{H}-\mathrm{NMR}\left(\mathrm{CDCl}_{3}, 200 \mathrm{MHz}\right) \delta 3.90(\mathrm{~s}, 3 \mathrm{H}$, $\left.\mathrm{OCH}_{3}\right), 4.78\left(\mathrm{dd}, J=4.4,1.4 \mathrm{~Hz}, 2 \mathrm{H}, \mathrm{OC}_{2} \mathrm{CH}=\mathrm{CHCO}\right), 6.44(\mathrm{dt}, J=11.6,1.4 \mathrm{~Hz}, 1 \mathrm{H}$, $\left.\mathrm{OCH}_{2} \mathrm{CH}=\mathrm{C} \underline{\mathrm{HCO}}\right), 6.76\left(\mathrm{dt}, J=11.6,4.4 \mathrm{~Hz}, 1 \mathrm{H}, \mathrm{OCH}_{2} \mathrm{C} \underline{\mathrm{H}}=\mathrm{CHCO}\right), 7.11(\mathrm{~m}, 2 \mathrm{H}, \mathrm{ArH}), 7.47$ $(\mathrm{dd}, J=6.6,3.2 \mathrm{~Hz}, 1 \mathrm{H}, \mathrm{ArH}) ;{ }^{13} \mathrm{C}-\mathrm{NMR}\left(\mathrm{CDCl}_{3}, 50 \mathrm{MHz}\right) \delta 56.3,69.3,116.4,121.9,123.8$, 131.8, 133.7, 141.9, 148.3, 151.4, 190.4; EI-MS (70 eV) $\mathrm{m} / z$ (rel. intensity, \%) $191\left([\mathrm{M}+1]^{+}, 44\right)$, $190\left(\mathrm{M}^{+}, 100\right), 161$ (32), 147 (12), 119 (13), 91 (27), 76 (12), 65 (13), 63 (15), 51 (18); HRMS calcd for $\mathrm{C}_{11} \mathrm{H}_{10} \mathrm{O}_{3}$ : 190.0630. Found: 190.0631 .

7-Bromo-2H-1-benzoxepin-5-one (5d). (1.95 g, 82 \%) was obtained as colorless crystal, mp 72$73^{\circ} \mathrm{C}, \mathrm{R}_{\mathrm{f}}=0.30$ (ethyl acetate: $n$-hexane $\left.=1: 9\right),{ }^{1} \mathrm{H}-\mathrm{NMR}\left(\mathrm{CDCl}_{3}, 400 \mathrm{MHz}\right) \delta 4.73(\mathrm{dd}, J=4.8$, $\left.1.2 \mathrm{~Hz}, 2 \mathrm{H}, \mathrm{OC}_{2} \mathrm{CH}=\mathrm{CHCO}\right), 6.41\left(\mathrm{dt}, J=11.6,1.2 \mathrm{~Hz}, 1 \mathrm{H}, \mathrm{OCH}_{2} \mathrm{CH}=\mathrm{C} \underline{\mathrm{HCO}}\right), 6.78(\mathrm{dt}, J=$ 11.6, $\left.4.8 \mathrm{~Hz}, 1 \mathrm{H}, \mathrm{OCH}_{2} \mathrm{C} \underline{\mathrm{H}}=\mathrm{CHCO}\right), 6.98(\mathrm{~d}, J=8.4 \mathrm{~Hz}, 1 \mathrm{H}, \mathrm{ArH}), 7.54(\mathrm{dd}, J=8.4,2.4 \mathrm{~Hz}, 1 \mathrm{H}$, $\operatorname{ArH}), 8.05(\mathrm{~d}, J=2.4 \mathrm{~Hz}, 1 \mathrm{H}, \mathrm{ArH}) ;{ }^{13} \mathrm{C}-\mathrm{NMR}\left(\mathrm{CDCl}_{3}, 100 \mathrm{MHz}\right) \delta 68.8,116.7,123.4,131.0$, 133.6, 133.9, 137.3 141.8, 157.9, 188.2; EI-MS (70 eV) $\mathrm{m} / \mathrm{z}$ (rel. intensity, \%) $240\left([\mathrm{M}+2]^{+}, 57\right)$, $238\left(\mathrm{M}^{+}, 57\right), 211$ (80), 209 (82), 131 (100), 103 (61), 102 (35), 77 (39), 63 (42); HRMS calcd for $\mathrm{C}_{10} \mathrm{H}_{7} \mathrm{BrO}_{2}$ : 237.9629. Found: 237.9630 .

7,9-Dichloro-2H-1-benzoxepin-5-one (5e). (1.91 g, 84\%) was obtained as colorless crystal, mp $111-112^{\circ} \mathrm{C}, \mathrm{R}_{\mathrm{f}}=0.39$ (ethyl acetate: $n$-hexane $\left.=1: 9\right),{ }^{1} \mathrm{H}-\mathrm{NMR}\left(\mathrm{CDCl}_{3}, 200 \mathrm{MHz}\right) \delta 4.81(\mathrm{dd}, J$ $\left.=4.6,1.4 \mathrm{~Hz}, 2 \mathrm{H}, \mathrm{OC}_{2} \mathrm{CH}=\mathrm{CHCO}\right), 6.41\left(\mathrm{dt}, J=11.8,1.4 \mathrm{~Hz}, 1 \mathrm{H}, \mathrm{OCH}_{2} \mathrm{CH}=\mathrm{C} \underline{\mathrm{HCO}}\right), 6.80$ $\left(\mathrm{dt}, J=11.8,4.6 \mathrm{~Hz}, 1 \mathrm{H}, \mathrm{OCH}_{2} \mathrm{C} \underline{\mathrm{H}}=\mathrm{CHCO}\right), 7.56(\mathrm{~d}, J=2.6 \mathrm{~Hz}, 1 \mathrm{H}, \mathrm{ArH}), 7.76(\mathrm{~d}, J=2.6 \mathrm{~Hz}$, $1 \mathrm{H}, \mathrm{ArH}) ;{ }^{13} \mathrm{C}-\mathrm{NMR}\left(\mathrm{CDCl}_{3}, 50 \mathrm{MHz}\right) \delta$ 69.4, 128.0, 129.1, 129.5, 132.7, 133.0, 134.2, 142.3, 152.9, 187.9; EI-MS (70 eV) m/z (rel. intensity, \%) $230\left([\mathrm{M}+2]^{+}, 42\right), 228\left(\mathrm{M}^{+}, 64\right), 201$ (68), 199 (100), 165 (33), 136 (21), 102 (31); HRMS calcd for $\mathrm{C}_{10} \mathrm{H}_{6} \mathrm{Cl}_{2} \mathrm{O}_{2}$ : 227.9745. Found: 227.9745 .

\section{General procedure for the preparation of 2,5-dihydro-1-benzoxepin-5-ol (6a-e)}

The (2-allyloxyphenyl)-2-propen-1-ol (3a-e) $(10 \mathrm{mmol})$ dissolved in anhydrous $\mathrm{CH}_{2} \mathrm{Cl}_{2}(100$ $\mathrm{mL}$ ) was stirred with added Grubbs' catalyst $(5 \mathrm{~mol} \%)$. The mixture was continually stirred at room temperature for $6 \mathrm{hr}$. Then, it was concentrated in vacuo to remove the solvent. The residue which was obtained was purified by silica gel column chromatography (ethyl acetate: $n$-hexane = 1: 15) to give pure 6a-e.

2,5-Dihydro-1-benzoxepin-5-ol (6a). ${ }^{4}(1.30 \mathrm{~g}, 80 \%)$ was obtained as colorless liquid, $\mathrm{R}_{\mathrm{f}}=0.18$ (ethyl acetate: $n$-hexane $=1: 10),{ }^{1} \mathrm{H}-\mathrm{NMR}\left(\mathrm{CDCl}_{3}, 400 \mathrm{MHz}\right) \delta 2.75(\mathrm{~d}, J=8.0 \mathrm{~Hz}, 1 \mathrm{H}, \mathrm{OH})$, 4.52 (ddd, $J=17.2,4.8,2.4 \mathrm{~Hz}, 1 \mathrm{H}, \mathrm{OCH}_{\mathrm{a}} \mathrm{H}_{\mathrm{b}} \mathrm{CH}=\mathrm{CH}$ ), 4.60 (ddd, $J=17.2,4.8,2.4 \mathrm{~Hz}, 1 \mathrm{H}$, $\left.\mathrm{OCH}_{\mathrm{a}} \underline{\mathrm{H}}_{\mathrm{b}} \mathrm{CH}=\mathrm{CH}\right), 5.48\left(\mathrm{~m}, 1 \mathrm{H}, \mathrm{OCH}_{2} \mathrm{C} \underline{\mathrm{H}}=\mathrm{CHCH}(\mathrm{OH})\right), 5.50\left(\mathrm{~m}, 1 \mathrm{H}, \mathrm{OCH}_{2} \mathrm{CH}=\mathrm{CHC} \underline{H}(\mathrm{OH})\right)$, 5.99 (ddt, $\left.J=11.6,4.0,2.4 \mathrm{~Hz}, 1 \mathrm{H}, \mathrm{OCH}_{2} \mathrm{CH}=\underline{\mathrm{CHCH}}(\mathrm{OH})\right), 7.08$ (m, 1H, ArH), 7.12 (m, 1H, 
$\mathrm{ArH}), 7.25$ (m, 1H, ArH), $7.32(\mathrm{~m}, 1 \mathrm{H}, \mathrm{ArH}) ;{ }^{13} \mathrm{C}-\mathrm{NMR}\left(\mathrm{CDCl}_{3}, 100 \mathrm{MHz}\right) \delta$ 68.9, 71.0, 121.6, 124.6, 125.4, 127.8, 128.9, 131.6, 139.2, 156.2; EI-MS (70 eV) m/z (rel. intensity, \%) $162\left(\mathrm{M}^{+}\right.$, 11), 145 (13), 144 (25), 133 (18), 132 (16), 131 (100), 115 (27), 105 (23), 77 (13); HRMS calcd for $\mathrm{C}_{10} \mathrm{H}_{10} \mathrm{O}_{2}$ : 162.0681. Found: 162.0679 .

8-Methoxy-2,5-dihydro-1-benzoxepin-5-ol (6b). (1.59 g, 83\%) was obtained as colorless liquids, $\mathrm{R}_{\mathrm{f}}=0.13$ (ethyl acetate: $n$-hexane $\left.=1: 9\right),{ }^{1} \mathrm{H}-\mathrm{NMR}\left(\mathrm{CDCl}_{3}, 200 \mathrm{MHz}\right) \delta 3.19(\mathrm{br}, 1 \mathrm{H}$, $\mathrm{OH}), 3.76\left(\mathrm{~s}, 3 \mathrm{H}, \mathrm{OCH}_{3}\right), 4.48\left(\mathrm{ddd}, J=17.2,4.4,2.2 \mathrm{~Hz}, 1 \mathrm{H}, \mathrm{OC}_{\mathrm{H}} \mathrm{H}_{\mathrm{b}} \mathrm{CH}=\mathrm{CH}\right), 4.57(\mathrm{ddd}, J=$ 17.2, 4.4, $\left.2.2 \mathrm{~Hz}, 1 \mathrm{H}, \mathrm{OCH}_{\mathrm{a}} \underline{\mathrm{H}}_{\mathrm{b}} \mathrm{CH}=\mathrm{CH}\right), 5.38\left(\mathrm{~m}, 1 \mathrm{H}, \mathrm{OCH}_{2} \mathrm{CH}=\mathrm{CHC} \underline{\mathrm{H}}(\mathrm{OH})\right), 5.46(\mathrm{~m}, 1 \mathrm{H}$, $\left.\mathrm{OCH}_{2}-\mathrm{C} \underline{\mathrm{H}}=\mathrm{CHCH}(\mathrm{OH})\right), 5.97\left(\mathrm{ddt}, J=11.6,4.2,2.2 \mathrm{~Hz}, 1 \mathrm{H}, \mathrm{OCH}_{2} \mathrm{CH}=\mathrm{CHCH}(\mathrm{OH})\right), 6.64(\mathrm{~d}$, $J=2.6 \mathrm{~Hz}, 1 \mathrm{H}, \mathrm{ArH}), 6.63(\mathrm{dd}, J=8.8,2.6 \mathrm{~Hz}, 1 \mathrm{H}, \mathrm{ArH}), 7.18(\mathrm{~d}, J=8.8 \mathrm{~Hz}, 1 \mathrm{H}, \mathrm{ArH}) ;{ }^{13} \mathrm{C}-$ NMR $\left(\mathrm{CDCl}_{3}, 50 \mathrm{MHz}\right) \delta 55.3,68.5,70.7,107.5,109.3,126.4,127.3,131.0,131.8,157.0$, 159.9; EI-MS (70 eV) m/z (rel. intensity, \%) 193 ([M+1] $\left.]^{+}, 13\right), 192\left(\mathrm{M}^{+}, 100\right), 177$ (29), 175 (37), 161 (45), 150 (80), 121 (29), 91 (47), 77 (42), 63 (39), 51 (35); HRMS calcd for $\mathrm{C}_{11} \mathrm{H}_{12} \mathrm{O}_{3}$ : 192.0786. Found: 192.0786.

9-Methoxy-2,5-dihydro-1-benzoxepin-5-ol (6c). (1.22 g, 64\%) was obtained as colorless crystal, mp $99-100^{\circ} \mathrm{C}, \mathrm{R}_{\mathrm{f}}=0.13$ (ethyl acetate: $n$-hexane $\left.=1: 10\right),{ }^{1} \mathrm{H}-\mathrm{NMR}\left(\mathrm{CDCl}_{3}, 400 \mathrm{MHz}\right.$ ) $\delta 2.81$ (br d, $J=8.0 \mathrm{~Hz}, 1 \mathrm{H}, \mathrm{OH}), 3.86\left(\mathrm{~s}, 3 \mathrm{H}, \mathrm{OCH}_{3}\right), 4.52$ (ddd, $J=17.6,4.8,2.4 \mathrm{~Hz}, 1 \mathrm{H}$, $\left.\mathrm{OCH}_{\mathrm{a}} \mathrm{H}_{\mathrm{b}} \mathrm{CH}=\mathrm{CH}\right), 4.59$ (ddd, $\left.J=17.6,4.8,2.4 \mathrm{~Hz}, 1 \mathrm{H}, \mathrm{OCH}_{\mathrm{a}} \underline{\mathrm{H}}_{b} \mathrm{CH}=\mathrm{CH}\right), 5.47(\mathrm{~m}, 1 \mathrm{H}$, $\left.\mathrm{OCH}_{2} \mathrm{C} \underline{\mathrm{H}}=\mathrm{CHCH}(\mathrm{OH})\right), 5.54\left(\mathrm{~m}, 1 \mathrm{H}, \mathrm{OCH}_{2} \mathrm{CH}=\mathrm{CHC} \underline{H}(\mathrm{OH})\right), 5.97$ (ddt, $J=11.6,4.4,2.4 \mathrm{~Hz}$, $1 \mathrm{H}, \mathrm{OCH}_{2} \mathrm{CH}=\mathrm{C} \underline{\mathrm{HCH}}(\mathrm{OH}), 6.88(\mathrm{dd}, J=8.0,1.6 \mathrm{~Hz}, 1 \mathrm{H}, \mathrm{ArH}), 6.93(\mathrm{dd}, J=8.0,1.6 \mathrm{~Hz}, 1 \mathrm{H}$, $\mathrm{ArH}), 7.07(\mathrm{t}, J=8.0 \mathrm{~Hz}, 1 \mathrm{H}, \mathrm{ArH}) ;{ }^{13} \mathrm{C}-\mathrm{NMR}\left(\mathrm{CDCl}_{3}, 100 \mathrm{MHz}\right) \delta 55.9,68.6,70.0,111.6$, 116.8, 124.8, 127.6, 131.6, 141.1, 144.0, 151.9; EI-MS (70 eV) m/z (rel. intensity, \%) 193 $\left([\mathrm{M}+1]^{+}, 34\right), 192\left(\mathrm{M}^{+}, 86\right), 175$ (27), 177 (19), 163 (34), 131 (36), 103 (100), 91 (49), 77 (38), 51 (24); HRMS calcd for $\mathrm{C}_{11} \mathrm{H}_{12} \mathrm{O}_{3}$ : 192.0786. Found: 192.0788.

7-Bromo-2,5-dihydro-1-benzoxepin-5-ol (6d). (1.86 g, 78\%) was obtained as colorless crystal, mp $111-113^{\circ} \mathrm{C}, \mathrm{R}_{\mathrm{f}}=0.20$ (ethyl acetate: $n$-hexane $\left.=1: 9\right),{ }^{1} \mathrm{H}-\mathrm{NMR}\left(\mathrm{CDCl}_{3}, 400 \mathrm{MHz}\right) \delta 2.58(\mathrm{~d}$, $J=7.6 \mathrm{~Hz}, 1 \mathrm{H}, \mathrm{OH}), 4.46\left(\mathrm{ddd}, J=17.2,4.8,2.4 \mathrm{~Hz}, 1 \mathrm{H}, \mathrm{OC}_{\mathrm{H}} \mathrm{H}_{\mathrm{b}} \mathrm{CH}=\mathrm{CH}\right), 4.63(\mathrm{ddd}, J=17.2$, 4.8, $\left.2.4 \mathrm{~Hz}, 1 \mathrm{H}, \mathrm{OCH}_{\mathrm{a}} \underline{\mathrm{H}}_{\mathrm{b}} \mathrm{CH}=\mathrm{CH}\right), 5.48\left(\mathrm{~m}, 1 \mathrm{H}, \mathrm{OCH}_{2} \underline{\mathrm{CH}}=\mathrm{CHCH}(\mathrm{OH})\right), 5.58(\mathrm{~m}, 1 \mathrm{H}$, $\left.\mathrm{OCH}_{2} \mathrm{CH}=\mathrm{CHC} \underline{\mathrm{H}}(\mathrm{OH})\right), 5.92\left(\mathrm{ddt}, J=12.0,3.6,2.4 \mathrm{~Hz}, 1 \mathrm{H}, \mathrm{OCH}_{2} \mathrm{CH}=\mathrm{C} \underline{\mathrm{HCH}}(\mathrm{OH})\right), 6.95(\mathrm{~d}, J$ $=8.4 \mathrm{~Hz}, 1 \mathrm{H}, \mathrm{ArH}), 7.35(\mathrm{dd}, J=8.4,2.4 \mathrm{~Hz}, 1 \mathrm{H}, \mathrm{ArH}), 7.49(\mathrm{~d}, J=2.4 \mathrm{~Hz}, 1 \mathrm{H}, \mathrm{ArH}) ;{ }^{13} \mathrm{C}-$ $\mathrm{NMR}\left(\mathrm{CDCl}_{3}, 100 \mathrm{MHz}\right) \delta 68.1,71.0,117.5,123.4,127.4,128.2,131.4,131.5,141.4,155.0$; EIMS (70 eV) m/z (rel. intensity, \%) 242 ([M+2] $\left.]^{+}, 17\right), 240\left(\mathrm{M}^{+}, 17\right), 211$ (46), 209 (44), 161 (24), 133 (45), 132 (100), 131 (33), 115 (59), 105 (23), 63 (22); HRMS calcd for $\mathrm{C}_{10} \mathrm{H}_{9} \mathrm{BrO}_{2}$ : 239.9786. Found: 239.9788 .

7,9-Dichloro-2,5-dihydro-1-benzoxepin-5-ol (6e). (1.95 g, 85\%) was obtained as colorless crystal, mp $139-140^{\circ} \mathrm{C}, \mathrm{R}_{\mathrm{f}}=0.26$ (ethyl acetate: $n$-hexane $\left.=1: 9\right),{ }^{1} \mathrm{H}-\mathrm{NMR}\left(\mathrm{CDCl}_{3}, 200 \mathrm{MHz}\right) \delta$ $2.53(\mathrm{~d}, J=7.4 \mathrm{~Hz}, 1 \mathrm{H}, \mathrm{OH}), 4.47$ (ddd, $\left.J=17.6,5.0,2.4 \mathrm{~Hz}, 1 \mathrm{H}, \mathrm{OC}_{\mathrm{H}_{\mathrm{a}}} \mathrm{H}_{\mathrm{b}} \mathrm{CH}=\mathrm{CH}\right), 4.70$ (ddd, $\left.J=17.6,5.0,2.4 \mathrm{~Hz}, 1 \mathrm{H}, \mathrm{OCH}_{\mathrm{a}} \underline{\mathrm{H}}_{\mathrm{b}} \mathrm{CH}=\mathrm{CH}\right), 5.46\left(\mathrm{~m}, 1 \mathrm{H}, \mathrm{OCH}_{2} \underline{\mathrm{C}}=\mathrm{CHCH}(\mathrm{OH})\right), 5.69(\mathrm{~m}, 1 \mathrm{H}$, $\left.\mathrm{OCH}_{2} \mathrm{CH}=\mathrm{CHC} \underline{H}(\mathrm{OH})\right), 5.89\left(\mathrm{ddt}, J=11.6,3.6,2.4 \mathrm{~Hz}, 1 \mathrm{H}, \mathrm{OCH}_{2} \mathrm{CH}=\mathrm{C} \underline{\mathrm{HCH}}(\mathrm{OH})\right), 7.27(\mathrm{~d}, J$ $=2.4 \mathrm{~Hz}, 1 \mathrm{H}, \mathrm{ArH}), 7.31(\mathrm{~d}, J=2.4 \mathrm{~Hz}, 1 \mathrm{H}, \mathrm{ArH}) ;{ }^{13} \mathrm{C}-\mathrm{NMR}\left(\mathrm{CDCl}_{3}, 50 \mathrm{MHz}\right) \delta$ 67.9, 69.9, 
$123.5,126.8,127.8,128.5,123.00,131.6,142.7,149.8$; EI-MS (70eV) $m / z$ (rel. intensity, \%) 232 $\left([\mathrm{M}+2]^{+}, 26\right), 230\left(\mathrm{M}^{+}, 41\right), 203$ (49), 201 (100), 199 (60), 189 (43), 167 (62), 166 (89), 149 (49), 133 (41), 132 (58), 131 (75); HRMS calcd for $\mathrm{C}_{10} \mathrm{H}_{8} \mathrm{Cl}_{2} \mathrm{O}_{2}$ : 229.9901. Found: 229.9903.

\section{Acknowledgements}

Financial support (Grant no., NSC-96-2113-M-037-006) from NSC, Taiwan is grateful.

\section{References}

1. Dumontet, V.; Thoison, O.; Omobuwajo, O. R.; Martin, M.-T.; Perromat, G.; Chiaroni, Angele; Riche, C.; Pais, M.; Sevenet, T.; Hadi, A. H. Tetrahedron 1996, 52, 6931.

2. (a) Tandon, V. K.; Singh, K. A.; Awasthi, A. K.; Khanna, J. M.; Lal, B.; Anand, N. Bioorg. \& Med. Chem. Lett. 2004, 14, 2867. (b) Lin, Y.-L.; Kuo, H.-S.; Wang, Y.-W.; Huang, S.-T. Tetrahedron 2003, 59, 1277. (c) Kaupmann, W.; Ohlendorf, H. W.; Wolf, K. U. Eur. J. Med. Chem. 1985, 20, 207. (d) Albrecht, G.; Ecker, G.; Tumova, I. Sci. Pharm. 1993, 61, 161. (e) Purcell, T.; Zard, L. Eur. Pat. Appl. 198,762, 1986 ; Chem. Abstr. 1987, 107, 198114. (f) Balme, G.; Coudanne, I.; Desbordes, P.; Huser, N.; Lemaire, P.; Mousques, A.; Nivlet, A.; Vors, J.-P. PCT Int. Appl. WO 2003014104, 2003 ; Chem. Abstr. 2003, 138, 187654. (g) Ryabukhin, S. V.; Volochnyuk, D. M.; Plaskon, A. S.; Naumchik, V. S.; Tolmachev, A. A. Synthesis 2007, 1214.

3. Hofmann, H.; Djafari, H. Z. Nat.forsch., B, J. Chem. Sci. 1989, 44, 220.

4. Tatsuoka, T.; Imao, K.; Suzuki, K.; Shibata, M.; Satoh, F.; Miyano, S.; Sumoto, K. Heterocycles 1990, 30, 749.

5. Hasegawa, E.; Tsuchida, H.; Tamura, M. Chem. Lett. 2005, 34, 1688.

6. Hofmann, H.; Djafari, H. Liebigs Ann. Chem. 1985, 3, 599.

7. von Angerer, S. Science of Synthesis 2004, 17, 653.

8. (a) Grubbs, R. H. Angew. Chem. Int. Ed. 2006, 45, 3760. (b) Briggs, T. F.; Dudley, G. B. Tetrahedron Lett. 2005, 46, 7793.

9. (a) Pospisil, J.; Potacek, M. Tetrahedron, 2007, 63, 337 and literature citied therein. (b) Orlek, B. S.; Sammes, P. G.; Weller, D. J. Tetrahedron 1993, 49, 8179.

10. 10.Nelson, W. L.; Burke, T. R., Jr. J. Med. Chem. 1979, 22, 1082.

11. Fuerstner, A.; Aiessa, C. J. Am. Chem. Soc. 2006, 128, 6306.

12. Campi, E. M.; Jackson, W. R.; McCubbin, Q. J.; Trnacek, A. E. Aust. J. Chem. 1996, 49, 219.

13. Lee, J. I.; Lee, H. S.; Kim, B. H. Synth. Commun. 1996, 26, 3201.

14. Martin, S. F.; Garrison, P. J. J. Org. Chem. 1982, 47, 1513. 